\title{
Problematic Instagram Use: The Role of Perceived Feeling of Presence and Escapism
}

\author{
Kagan Kircaburun ${ }^{1}$ - Mark D. Griffiths ${ }^{2}$
}

Published online: 4 June 2018

(C) The Author(s) 2018

\begin{abstract}
The use of social networking sites is becoming increasingly popular. Although there are many studies investigating the problematic use of social networking sites such as Facebook, little is known about problematic Instagram use (PIU) and factors related to it. The present study developed a complex model in order to examine the mediating role of perceived feeling of presence (i.e., social, spatial, and co-presence) and escapism between using different Instagram features and PIU. A total of 333 Instagram users from a high school and a state university, aged between 14 and 23 years ( $M_{\text {age }}=17.74$ years, $\mathrm{SD}=2.37,61 \%$ female), completed a "paper-andpencil" questionnaire comprising measures of social presence, spatial presence, co-presence, Instagram escapism, and PIU. In addition, frequency of use of five different Instagram features (i.e., watching live streams; watching videos; looking at posted photographs; liking, commenting on others' posts; and getting likes and comments from others) were assessed using a 7-point Likert scale. Analysis indicated that watching live streams was indirectly associated with PIU via escapism, spatial presence, and co-presence. Leaving likes and comments on others' posts was both directly and indirectly associated with PIU via co-presence and escapism. Escapism mediated the relationships between social and spatial presence and co-presence and PIU. The findings of the present study appear to indicate that a minority of individuals use Instagram problematically and that problematic Instagram use is associated with the frequency of watching live streams, liking, and commenting on others' posts on Instagram, being able to feel a higher sense of presence using Instagram, and using Instagram as an escape from reality.
\end{abstract}

Keywords Problematic Instagram use $\cdot$ Social presence $\cdot$ Spatial presence $\cdot$ Co-presence $\cdot$ Escapism

Mark D. Griffiths

mark.griffiths@ntu.ac.uk

1 Faculty of Education, Duzce University, Duzce, Turkey

2 International Gaming Research Unit, Psychology Department, Nottingham Trent University, 50

Shakespeare Street, Nottingham NG1 4FQ, UK 
The number of individuals that now use Instagram across the world is approximately 800 million monthly and 500 million daily active users (Aslam 2018). Approximately 95 million photographs and 300 million live streams are uploaded on a daily basis, and these posts receive 4.2 billion "likes" daily (Aslam 2018). Instagram use is expanding and surpassing other social networking platforms such as Facebook, Twitter, and Snapchat especially among adolescents (Bahtiyar and Kircaburun 2017) and emerging adults (Kircaburun and Bahtiyar 2017). This popularity brings about risks of becoming problematic for some of its users (Kuss and Griffiths 2017).

Over the last decade, related factors and consequences of problematic social networking site use have been of increasing concern and empirical study (Kuss and Griffiths 2017). While some of these studies have focused on the use of social media more generally (Bányai et al. 2017; Kırcaburun 2016a; Kircaburun, Alhabash, Tosuntaş, \& Griffiths, 2018a; Kircaburun, Demetrovics, \& Tosuntaş, 2018b; Kircaburun et al., 2018c; Monacis et al. 2017; Wegmann et al. 2015), some studies have specifically investigated problematic and addictive use of specific social networking sites such as Facebook (Satici 2018; Young et al. 2017), Twitter (Kurcaburun 2016b), and YouTube (Balakrishnan and Griffiths 2017). Although there is no official diagnostic system (e.g., DSM-5; American Psychiatric Association 2013) or widely accepted diagnostic criteria (Spada 2014) for social media disorder, problematic social media use - which is a specific type of problematic Internet use (Griffiths et al. 2014) - has been conceptualized as a potential behavioral addiction within a biopsychosocial framework comprising core addiction components (Griffiths 2005). These include withdrawal (e.g., becoming irritable when not using social media), conflict (e.g., experiencing negative consequences of social media use in real life), tolerance (e.g., need to spend increasingly more time engaged in social media activities), salience (e.g., spending lots of time and being totally preoccupied with social media), mood modification (e.g., using social media to modify mood, e.g., to forget about problems), and relapse (e.g., unsuccessful attempts to stop engaging in social media use). The use of these criteria has led to the development of psychometrically robust instruments to assess social media addiction (Andreassen et al. 2016).

Even though the problematic use of social media in general as well as specific platforms such as Facebook has been the topic of numerous studies, very little is known about problematic Instagram use (PIU) (Kircaburun \& Griffiths, 2018). Despite the fact that social media platforms have become increasingly similar adopting similar features, each platform has unique features, has a characteristic structure, and has different motives and gratifications underlying their use (Alhabash and Ma 2017). Consequently, there is a need for PIU to be investigated alongside potential factors that might be associated with it. Based on empirical evidence, previous studies have suggested that increased feelings of presence and escapism are associated with addictive use of Internet applications such as online gaming and social media (Gao et al. 2017; Park and Hwang 2009). Consequently, the purpose of the present study was to develop a complex model in which (i) Instagram features were independent variables (i.e., watching live streams and videos, looking at others' photographs, liking or commenting on others' posts, and others' likes and comments' on their posts), (ii) different types of presence that are felt by Instagram users (i.e., social, spatial, and co-presence) and Instagram escapism were mediator variables, and (iii) PIU was the outcome variable.

\section{Problematic Instagram Use and Use of Different Instagram Features}

Instagram comprises widely used features such as video and photo sharing, and liking or commenting on others' posts. More recently, Instagram provided its users with the capability 
to create live stories and to share them with followers. Although, to date, there is no study that has reported on how different Instagram features are related to problematic use, it has been shown that the frequent use of Likes and Wall updates on Facebook is related to bonding social capital (Lee et al. 2014), and that using different Facebook features (e.g., likes, shares, comments, and status updates) has different psychophysiological effects on individuals (Alhabash et al. 2018). Moreover, Chen (2011) found that tweeting, retweeting, and replying to others' tweets more frequently were positively associated with the gratification of the need to connect to others on Twitter. These studies indicate that using different features of social media sites is associated with social gratifications and feelings of higher social connectedness (Chen 2011). Other studies have found that seeking friendship and relationship maintenance motives and gratifications are associated with more frequent social media use and social media addiction (Chen and Kim 2013; Huang 2011). Additionally, given that individuals' fear of missing out recent posts and topics on social media have been found to be associated with social media addiction (Blackwell et al. 2017), watching live streams and following recent posts of others and the urge to give quick feedback (e.g., liking or commenting) on these posts may contribute to problematic use. Therefore, it was hypothesized in the present study that using different Instagram features would be associated with PIU.

\section{Problematic Instagram Use and Perceived Feeling of Presence}

Presence has been defined as a perceptual illusion of non-mediation in which medium appears to become either invisible, or transformed into a social entity (Lombard and Ditton 1997), and has been recognized and examined under via interrelated sub-types (i.e., social, spatial, and copresence) by some researchers (e.g., Ijsselsteijn et al. 2000; Slater et al. 2000).

Social presence theory, which has been defined as "the degree of salience of the other person in the interaction and the consequent salience of the interpersonal relationships" (Short et al. 1976; p. 65), refers to the perceived feeling of existence in a specific medium as a result of an evaluation of social interactions with others in that particular platform (Heeter 1992). The more individuals feel others' existence in a virtual environment the more they feel attached to it, and this can lead to higher sense of belonging in the environment by increasing human warmth and contact (Gao et al. 2017). As mentioned earlier, individuals use different features of social media sites with social motivations in order to bond with others. Consequently, using these features may lead to a higher feeling of social presence. Although, no previous study has ever examined the direct relationship between social presence and PIU, a few studies have demonstrated that social presence may be associated with social media site addiction via escapism (Gao et al. 2017).

Spatial presence is the sense of being located in a mediated space, feeling surrounded by the media environment, and losing the sense of awareness toward the technological source of the experience (Hartmann et al. 2015). Spatial presence theory has been widely used to understand technology-mediated communication such as virtual reality (Coxon et al. 2016; Westerman et al. 2015), video gaming (Murphy 2016; Williams 2014), and educational settings (Stavropoulos et al. 2017). Bracken (2005) showed that watching high-definition television was associated with higher sense of spatial presence, and that higher sense of spatial presence is positively affected by higher emotional involvement with the consumed media (Wirth et al. 2012). Contemporary smartphones have high-definition screens and this increased functionality benefits Instagram users who follow other users (e.g., friends and celebrities) that draw 
their interest (higher emotional involvement) because they can watch live streams and videos of the individuals they follow. Using different features of Instagram is likely to give its users a sense of spatial presence, and it is hypothesized that a higher sense of spatial presence should lead to higher involvement in Instagram use because increased sense of spatial presence in a specific medium leads to higher enjoyment obtained in that medium (Hartmann et al. 2010) and can lead to addictive use in a small minority of individuals (Gao et al. 2017).

Co-presence, which has been referred as "being there together with other geographically dispersed users" (Dalgarno and Lee 2010; p. 14), is considered as the intersection between social and spatial presence (Ling et al. 2014). In a more recent definition, Ling et al. (2014) referred to co-presence as "the feeling of being together with others in a computer-generated world at the same time even though people are in separate places" (p. 2). It has been found that co-presence in virtual worlds increases user satisfaction and immersive tendencies (Bulu 2012). Moreover, Greitemeyer et al. (2014) found that having responsive Facebook friends was associated with the satisfaction of psychological needs more than the number of Facebook friends. Being able to interact with others in the medium by mutually liking and commenting on each other's posts increases the feeling of enjoyment and may lead to positive mood modification, which in a small minority of users may result in more problematic use of that particular medium (Gao et al. 2017). Consequently, based on the findings of the aforementioned studies, it was hypothesized that social, spatial, and co-presence would be directly associated with PIU and would mediate between use of Instagram features and PIU.

\section{Problematic Instagram Use and Escapism}

Escape theory-originally suggested as an explanation for suicidal behaviors (Baumeister 1990) - asserts that individuals who have higher standards and ideals than they can ever actually obtain are more likely to experience a need to escape from themselves or their reality (Lee 2017). Young et al. (2017) defined escapism as "a behavior employed to distract oneself from real life problems" (p. 25). These problems may be psychological ones such as depression, anxiety, and low self-esteem (Lee 2017). Several studies suggest that engaging in online activities such as playing online videogames and social media use to escape from reality, for distraction, or as a coping strategy is associated with problematic use (Gao et al. 2017; Masur et al. 2014; Yee 2006).

As well as real-life problems, individuals sometimes feel the need to escape from their reality because of a lack of things to do and this can act as either as an escape from real-life troubles or to avoid thinking about unpleasant thoughts (Hartmann et al. 2010). In order to successfully escape from reality, individuals need to have the feeling of involvement in the media, and therefore, they need to have the feeling of presence when using the media (Hartmann et al. 2010). In order to escape from themselves by feeling present in Instagram, individuals need to use different features of Instagram such as watching others' videos and live streams, looking at their photos, interacting with other users by liking or commenting on others' posts, and vice versa. As previously suggested (Gao et al. 2017), a small minority of individuals that successfully escape from reality by using Instagram may develop PIU because it helps modify their mood, one of one of the dimensions for problematic use if combined with other negative components such as conflict (Griffiths 2005). Based on the existing literature, it was hypothesized that escapism would be positively associated with PIU and would mediate between use of different Instagram features, feeling of presence, and PIU. 


\section{Methods}

\section{Participants}

A purposive sample of 333 Instagram users, aged between 14 and 23 years $\left(M_{\text {age }}=\right.$ 17.74 years, $\mathrm{SD}=2.37,61 \%$ female) participated in the study. Participants were recruited from a high school and a state university in northern region of Turkey and completed a "paper-and-pencil" questionnaire. Inclusion criterion for participation in the study was to have an active Instagram account.

\section{Measures}

The questionnaire comprised (i) basic sociodemographic questions, (ii) questions about the use of five Instagram features (details below), and (iii) four psychometric scales-Instagram Use Questionnaire, Social Presence Scale, Spatial Presence Experience Scale, and Escapism Scale (details below; total scale scores for all four scales were summative and the score ranges for each scale are listed in Table 1).

Use of Instagram Features In order to assess the use of different Instagram features, participants were asked to respond to five items regarding their use of (i) live streams ("I watch others' live streams on Instagram"), (ii) videos ("I watch others' videos on Instagram"), (iii) photos ("I look at others' photos on Instagram"), (iv) leaving likes and comments on others' posts ("I leave likes and comments on others' posts on Instagram"), and (v) others' likes and comments to their posts ("Others leave likes and comments on my posts on Instagram"), on a 7-point Likert scale from "absolutely not true" to "absolutely true."

Instagram Use Questionnaire (IUQ) The Social Media Use Questionnaire (SMUQ; Xanidis and Brignell 2016) assesses problematic social media use across nine items (e.g., "I

Table 1 Mean scores, standard deviations, score ranges, and Pearson correlation coefficients of the study variables

\begin{tabular}{|c|c|c|c|c|c|c|c|c|c|c|}
\hline & 1. & 2. & 3. & 4. & 5. & 6. & 7. & 8. & 9. & 10. \\
\hline $\begin{array}{l}\text { 1. Problematic Instagram } \\
\text { use }\end{array}$ & - & & & & & & & & & \\
\hline 2. Escapism & $.57 * * *$ & - & & & & & & & & \\
\hline 3. Social presence & $.42 * * *$ & $.42 * * *$ & - & & & & & & & \\
\hline 4. Spatial presence & $.32 * * *$ & $.38 * * *$ & $.40 * * *$ & - & & & & & & \\
\hline 5. Co-presence & $.48 * * *$ & $.53 * * *$ & $.56 * * *$ & $.34 * * *$ & - & & & & & \\
\hline 6. Watching live streams & $.25 * * *$ & $.21 * * *$ & $.39 * * *$ & $.28 * * *$ & $.42 * * *$ & - & & & & \\
\hline 7. Watching videos & $.30 * * *$ & $.34 * * *$ & $.39 * * *$ & $.19 * *$ & $.37 * * *$ & $.49 * * *$ & - & & & \\
\hline $\begin{array}{l}\text { 8. Looking at } \\
\text { photographs }\end{array}$ & $.28 * * *$ & $.33 * * *$ & $.44 * * *$ & $.20 * * *$ & $.37 * * *$ & $.33 * * *$ & $.63 * * *$ & - & & \\
\hline 9. $\mathrm{LCO}$ & $.38 * * *$ & $.34 * * *$ & $.40 * * *$ & $.14 *$ & $.45 * * *$ & $.25 * * *$ & $.48 * * *$ & $.62 * * *$ & - & \\
\hline 10. OLC & $.28 * * *$ & $.24 * * *$ & $.40 * * *$ & .10 & $.42 * * *$ & $.25 * * *$ & $.43 * * *$ & $.59 * * *$ & $.60 * * *$ & - \\
\hline Mean & 22.23 & 12.86 & 21.55 & 10.57 & 9.87 & 3.58 & 5.01 & 5.67 & 4.93 & 5.26 \\
\hline $\mathrm{SD}$ & 8.12 & 6.70 & 6.09 & 6.31 & 3.85 & 1.91 & 1.57 & 1.48 & 1.69 & 1.66 \\
\hline Score ranges & $9-45$ & $4-28$ & $5-35$ & $4-28$ & $3-21$ & $1-7$ & $1-7$ & $1-7$ & $1-7$ & $1-7$ \\
\hline
\end{tabular}

$L C O$ liking, commenting on others' posts; $O L C$ others like and comment on my posts

$* * * p<.001, * * p<.01, * p<.05$ 
feel anxious, when I am not able to check my social network account") on a 5-point Likert scale from "never" to "always," comprising two factors (withdrawal and compulsion). Previous studies have reported optimal validity and reliability of the scale (Kircaburun et al., 2018a). In the present study, in order to assess problematic Instagram use, the SMUQ was used and replaced the words "social media" with "Instagram." Because of this modification, confirmatory factor analysis (CFA) was carried out. CFA results $\left(\chi^{2} / \mathrm{df}=3.38\right.$, RMSEA $=.08$ (CI 90\% [.06, .10]), SRMR $=.02, \mathrm{CFI}=.97, \mathrm{GFI}=.95$ ) confirmed the two-dimensional structure and that the new IUQ was valid and reliable for assessing problematic Instagram use of adolescents and emerging adults. Internal validity of the scale was high (.89).

Social Presence Scale (SPS) The SPS (Gao et al. 2017) unidimensionally assesses levels of social presence and involvement felt by an individual while using social media, and comprises five items (e.g., "There is a sense of human contact in Instagram.", "There is a sense of sociability in Instagram.") on a 7-point Likert scale from "absolutely not true" to "absolutely true." The Turkish form of the scale was used for the first time in the present study. Therefore, confirmatory factor analysis (CFA) was applied. According to CFA results $\left(\chi^{2} / \mathrm{df}=.71\right.$, $\mathrm{RMSEA}=.00(\mathrm{CI} 90 \%[.00, .06]), \mathrm{SRMR}=.02, \mathrm{CFI}=1.00, \mathrm{GFI}=1.00)$, the scale was valid and reliable for assessing social presence levels felt in Instagram by Turkish participants. Internal validity of the scale was adequate (.74).

Spatial Presence Experience Scale (SPES) The SPES (Hartmann et al. 2015) assesses levels of spatial (place) presence and physical involvement felt by an individual while listening to a presentation and comprised four modified items (e.g., "I feel like I am actually there in the environment of the live streams and videos when I watch on Instagram.", "I feel like I am physically present in the environment of the live streams and videos when I watch on Instagram.") on a 7-point Likert scale from "absolutely not true" to "absolutely true." The Turkish form of the scale was used for the first time in the present study. Therefore, a CFA was carried out. According to CFA results $\left(\chi^{2} / \mathrm{df}=1.69\right.$, $\mathrm{RMSEA}=.04(\mathrm{CI} 90 \%[.00, .16]), \mathrm{SRMR}=.00, \mathrm{CFI}=1.00, \mathrm{GFI}=1.00)$, the scale was valid and reliable for assessing spatial presence levels felt in Instagram by Turkish participants. Internal validity of the scale was excellent (.92).

Co-presence Scale (CPS) The CPS (Poeschl and Doering 2015) assesses levels of copresence felt by an individual while in a virtual room comprising three modified items (e.g., "I don't feel alone while I am wandering in Instagram.", "I feel like I am connected to other users when I am in Instagram.") on a 7-point Likert scale from "absolutely not true" to "absolutely true." The Turkish form of the scale was used for the first time in the present study. Therefore, a CFA was carried out. According to CFA results $\left(\chi^{2} / \mathrm{df}=0.00, \mathrm{RMSEA}=.00(\mathrm{CI}\right.$ $90 \%[.00, .00]), \mathrm{SRMR}=.00, \mathrm{CFI}=1.00, \mathrm{GFI}=1.00)$, the scale was valid and reliable for assessing co-presence levels felt in Instagram by Turkish participants. The internal validity of the scale was adequate (.71). Moreover, analysis $\left(\chi^{2} / \mathrm{df}=1.56, \mathrm{RMSEA}=.04\right.$ (CI 90\% [.02, $.06]), \mathrm{SRMR}=.05, \mathrm{CFI}=.98, \mathrm{GFI}=.96)$ also indicated that the social, spatial, and co-presence scales can be used together as a unidimensional presence scale $(\alpha=.86)$.

Escapism Scale (ES) The ES (Gao et al. 2017) assesses the feeling of escape from real world felt by an individual while using Instagram comprising four modified items (e.g., "Instagram helps me escape from the world of reality.", "Instagram helps me escape from problems and 
pressures.") on a 7-point Likert scale from "absolutely not true" to "absolutely true." Turkish form of the scale was used for the first time in the present study. Therefore, a CFA was carried out. CFA results $\left(\chi^{2} / \mathrm{df}=1.52, \mathrm{RMSEA}=.04(\mathrm{CI} 90 \%[.00, .12]), \mathrm{SRMR}=.01, \mathrm{CFI}=1.00\right.$, $\mathrm{GFI}=1.00$ ) confirmed the unidimensional structure and that the scale was valid and reliable for assessing escapism levels felt in Instagram by Turkish participants. The internal validity of the scale was high (.89).

\section{Ethics}

The study was approved by the first author's university ethics committee and was carried out in accordance with the 1975 Helsinki declaration. All of the participants have given their informed consent and participated in the study voluntarily and anonymously.

\section{Statistical Analysis}

In order to analyze the data, Pearson correlation tests, confirmatory factor analyses (CFAs), and path analyses were applied via using SPSS 23.0 and AMOS 23.0 software. In the CFA and path analyses, goodness of fit criteria determined by $\mathrm{Hu}$ and Bentler (1999) were used in order to designate model fit. Consequently, thresholds for good and acceptable fit values are as follows: root mean square residuals (RMSEA) $<.05$ is good, standardized root mean square residuals (SRMR) $<.05$ is good, comparative fit index $(\mathrm{CFI})>.95$ is good, normed fit index $(\mathrm{NFI})>.95$ is good, goodness of fit index $(\mathrm{GFI})>.95$ is good, also RMSEA $<.08$ is acceptable, SRMR $<.08$ is acceptable, CFI $>$ .90 is acceptable, NFI $>.90$ is acceptable, and GFI $>.90$ is acceptable. Total, direct, and indirect effects of independent and mediator variables on outcome variable were calculated via using bootstrapping method with 5000 bootstrap samples and $95 \%$ biascorrected confidence intervals.

\section{Results}

Mean scores, standard deviations, score ranges, and Pearson correlation coefficients are presented in Table 1. Path analysis with the hypothesized model (Fig. 1) suggested good fit to the data $\left(\chi^{2} / \mathrm{df}=2.07, \mathrm{RMSEA}=.06(\mathrm{CI} 90 \%[.01, .10]), \mathrm{SRMR}=.01, \mathrm{CFI}=.99, \mathrm{GFI}=\right.$ .99). In the model, all variables except problematic Instagram use (PIU) were observed variables and PIU was a latent variable. Watching live streams was indirectly associated with PIU via escapism, spatial presence, and co-presence $(\beta=.12, p<01 ; 95 \%$ CI $[.04, .20])$. Leaving likes and comments on others' posts, on the other hand, was both directly $(\beta=.16$, $p<01 ; 95 \% \mathrm{CI}[.04, .29])$ and indirectly associated with PIU via co-presence and escapism $(\beta=.14, p<01 ; 95 \%$ CI $[.06, .23])$.

Spatial presence was weakly indirectly associated with PIU via escapism $(\beta=.08, p<001$; $95 \%$ CI $[.03, .15])$, meanwhile co-presence was directly $(\beta=.18, p<05 ; 95 \%$ CI $[.02, .33])$ and indirectly related to PIU via escapism $(\beta=.16, p<001 ; 95 \%$ CI $[.10, .23])$. Direct and indirect effects of social presence on PIU were insignificant, but its total effect on PIU was significant $(\beta=.15, p<05 ; 95 \%$ CI $[.02, .29]$; Table 2$)$, albeit weak. Total effect sizes of leaving likes and comments on others' posts and co-presence were moderate, whereas 


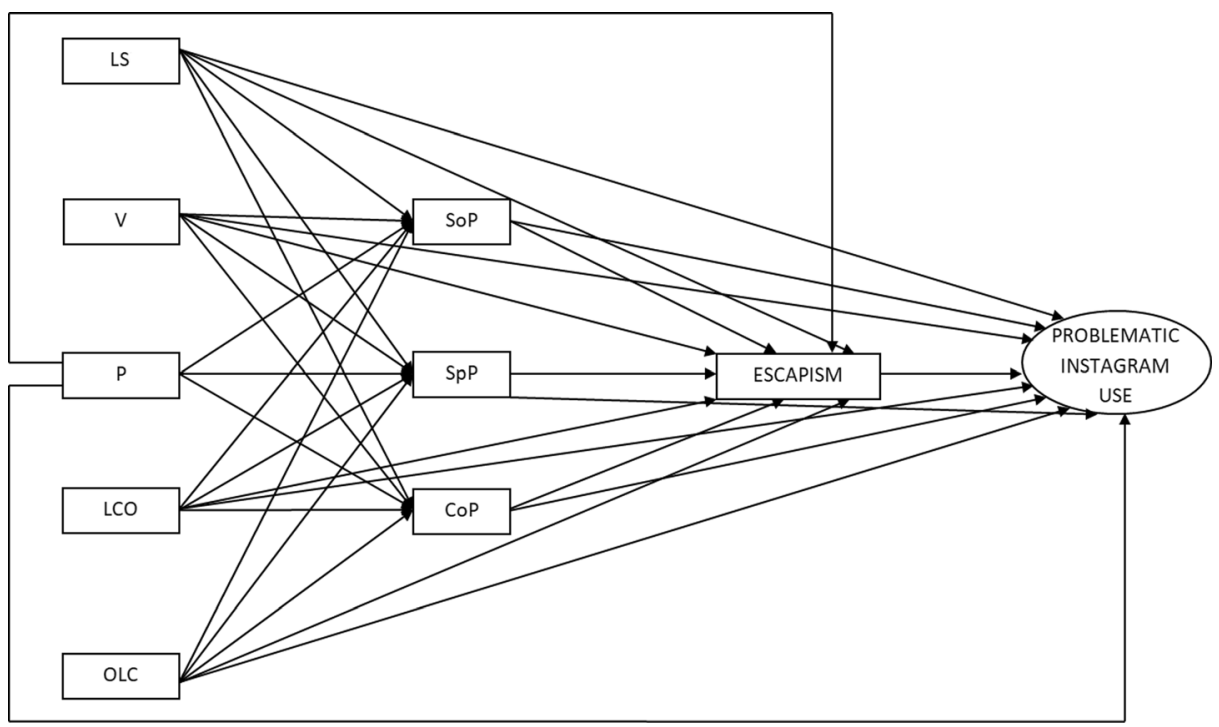

Fig. 1 Hypothesized model. For clarity, correlations between the independent and mediator variables have not been depicted in the figure. $L S$ watching live streams; $V$ watching videos; $P$ looking at photos; $L C O$ liking, commenting on others' posts; $O L C$ others' likes and comments on my posts; $S o P$ social presence; $S p P$ spatial presence; $C o P$ co-presence

watching live streams, social presence, and spatial presence were weak. The final model explained $49 \%$ of the variance in PIU (Fig. 2).

\section{Discussion}

The present study used presence and escape theories to develop a complex model examining the relationships between use of different Instagram features, different types of presence (i.e., social, spatial, and co-presence), escapism, and problematic Instagram use (PIU). Consequently, results suggest that watching live streams (LS) and liking and commenting on others' posts (LCO) in Instagram, higher feeling of presence, and escapism were associated with PIU.

Partially in line with the hypotheses, LS and LCO were associated with PIU. The relationship between LS and PIU was more complex. While higher LS was negatively indirectly associated with PIU via escapism, it was positively indirectly associated with PIU via spatial presence and co-presence, and the overall effect was positive. This may be explained if Instagram users were watching live streams for another reason than to escape from their reality (e.g., fear of missing out the recent updates of their followed ones). However, since watching live streams was giving individuals a higher feeling of spatial presence and co-presence, this led to a higher feeling of escape, and in turn, higher PIU scores. On the other hand, LCO was both directly and indirectly associated with PIU via co-presence and escapism. Similar to LS, the direct effect of LCO may be explained by social gratifications such as higher bonding social capital (Lee et al. 2014) and fear of missing out the recent updates (Blackwell et al. 2017). These users may have become problematic Instagram users with the urge to give instant and quick feedback on the posts of individuals they followed out of their need for more engaged social interactions. Moreover, those individuals who left more likes and comments on 
Table 2 Standardized estimates of total, direct, and indirect effects on problematic Instagram use and mediator variables

\begin{tabular}{|c|c|c|c|}
\hline & Effect & S.E. & $\%$ explained of total effect \\
\hline LS $\rightarrow$ PIU (total effect) & $.15^{*}$ & .07 & - \\
\hline $\mathrm{LS} \rightarrow \mathrm{PIU}$ (direct effect) & .03 & .06 & 20 \\
\hline $\mathrm{LS} \rightarrow \mathrm{PIU}$ (total indirect effect) & $.12 * *$ & .04 & 80 \\
\hline $\mathrm{LS} \rightarrow \mathrm{CoP} \rightarrow \mathrm{PIU}$ & $.05 *$ & .06 & 33 \\
\hline $\mathrm{LS} \rightarrow$ escapism $\rightarrow$ PIU & $-.06 * *$ & .05 & 40 \\
\hline $\mathrm{LS} \rightarrow \mathrm{SpP} \rightarrow$ escapism $\rightarrow$ PIU & $.02 * * *$ & .02 & 13 \\
\hline $\mathrm{LS} \rightarrow \mathrm{CoP} \rightarrow$ escapism $\rightarrow$ PIU & $.05 * * *$ & .03 & 33 \\
\hline $\mathrm{LCO} \rightarrow$ PIU (total effect) & $.31 * * *$ & .07 & - \\
\hline $\mathrm{LCO} \rightarrow \mathrm{PIU}$ (direct effect) & $.16^{* *}$ & .06 & 54 \\
\hline $\mathrm{LCO} \rightarrow$ PIU (total indirect effect) & $.14 * *$ & .04 & 46 \\
\hline $\mathrm{LCO} \rightarrow \mathrm{CoP} \rightarrow \mathrm{PIU}$ & $.04 *$ & .06 & 14 \\
\hline $\mathrm{LCO} \rightarrow \mathrm{CoP} \rightarrow$ escapism $\rightarrow$ PIU & $.04 * * *$ & .03 & 13 \\
\hline SoP $\rightarrow$ PIU (total effect) & $.15^{*}$ & .07 & - \\
\hline SoP $\rightarrow$ PIU (direct effect) & .11 & .07 & 73 \\
\hline SoP $\rightarrow$ escapism $\rightarrow$ PIU (total indirect effect) & .04 & .02 & 27 \\
\hline $\mathrm{SpP} \rightarrow \mathrm{PIU}$ (total effect) & $.16^{*}$ & .06 & - \\
\hline $\mathrm{SpP} \rightarrow \mathrm{PIU}$ (direct effect) & .08 & .06 & 50 \\
\hline $\mathrm{SpP} \rightarrow$ escapism $\rightarrow$ PIU (total indirect effect) & $.08^{* *}$ & .03 & 50 \\
\hline $\mathrm{CoP} \rightarrow \mathrm{PIU}$ (total effect) & $.34 * *$ & .08 & - \\
\hline $\mathrm{CoP} \rightarrow \mathrm{PIU}$ (direct effect) & $.18^{*}$ & .08 & 53 \\
\hline $\mathrm{CoP} \rightarrow$ escapism $\rightarrow$ PIU (total indirect effect) & $.16^{* * * *}$ & .03 & 47 \\
\hline
\end{tabular}

Only significant indirect effects are shown in the table

$L S$ watching live streams; $L C O$ liking, commenting on others' posts; $S o P$ social presence; $S p P$ spatial presence; CoP co-presence; $P I U$ problematic Instagram use

$* * * p<.001, * * p<.01, * p<.05$

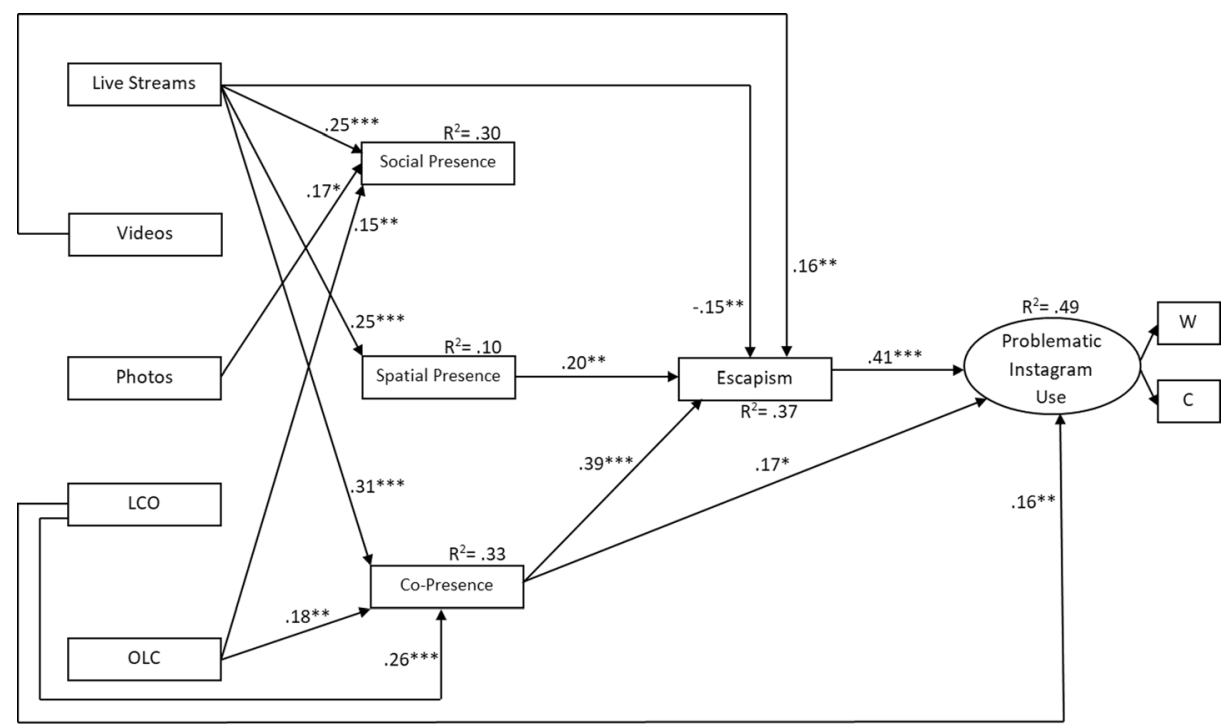

Fig. 2 Final model of the significant standardized path coefficients between variables. For clarity, correlations between the independent variables, mediator variables, and insignificant path coefficients have not been depicted in the figure. $L C O$ liking, commenting on others' posts; $O L C$ others' likes and comments on my posts; $W$ withdrawal; $C$ compulsion. $* * * p<.001, * * p<.01, * p<.05$ 
others' posts experienced a higher feeling of co-presence, and this was another factor that led to higher feeling of escape from reality, thereby to higher PIU scores. This result supports the study of Young et al. (2017) who found that passive Facebook use was not associated with escapism. In order to obtain a sense of escape, users need to be interacting with other users actively. Consequently, using two of the five Instagram features was directly or indirectly associated with higher PIU scores.

With regard to feeling of presence, partially parallel to our hypotheses and previous studies (Gao et al. 2017), all three types of presence had significant total effects on PIU. However, direct and indirect effects of social presence were not significant. The relationship between spatial presence and PIU was fully explained by escapism. Instagram use was leading its users to feel higher spatial presence and this led to higher feeling of escapism. It seems that users feel higher emotional involvement toward videos they watch in Instagram that they feel higher spatial presence (Wirth et al. 2012). This may be because users follow other users that they feel emotionally attached to, such as close friends, family, or popular celebrities. Watching videos of others that they feel emotionally involved help them escape from their unpleasant reality and this positive mood modification leads them to higher PIU scores. Moreover, feeling of being together with others (i.e., co-presence) led to higher PIU scores via increased feeling of escape from themselves. Individuals that have a small number of real life friends may need to compensate their social needs in virtual platforms. Finding others via Instagram that can provide mutual interactions may fulfill individuals' social needs and help them escape from their reality where they feel lonely. The urge to repeatedly experience such social gratifications may canalize individuals to develop a sense of belonging to the platform and this may lead to problematic use for a minority of individuals (Gao et al. 2017).

The present study is not without limitations. The sample was cross-sectional and nonrepresentative; consequently, the results are unable to indicate any causal relationships between variables. Longitudinal studies are needed in order to demonstrate causal relationships between all the variables in the present study. Data concerning the feeling of presence, escapism, and PIU were collected using self-report questionnaires; therefore, several biases may be affecting the results such as social desirability and memory recall. Future studies could use qualitative or mixed methods in order to collect more triangulated data for more reliable in-depth results.

Despite its limitations, the present study has also has several strong aspects. This study is one of the first to examine problematic use of Instagram and the first to investigate how use of different Instagram features (i.e., watching live streams and videos, looking at photos, liking and commenting on others' posts, receiving likes and comments from others) affect individuals' sense of presence, escapism, and PIU scores. The findings of the present study appear to indicate that a minority of individuals use Instagram problematically and problematic Instagram use is associated with the frequency of watching live streams, liking and commenting on others' posts on Instagram, being able to feel a higher sense of presence using Instagram, and using Instagram as an escape from reality.

\section{Compliance with Ethical Standards}

Conflict of Interest The authors declare that they have no conflict of interest. 
Open Access This article is distributed under the terms of the Creative Commons Attribution 4.0 International License (http://creativecommons.org/licenses/by/4.0/), which permits unrestricted use, distribution, and reproduction in any medium, provided you give appropriate credit to the original author(s) and the source, provide a link to the Creative Commons license, and indicate if changes were made.

\section{References}

Alhabash, S., Almutairi, N., Lou, C., \& Kim, W. (2018). Pathways to virality: Psychophysiological responses preceding likes, shares, comments and status updates on Facebook. Media Psychology, 2018, 1-21. https://doi.org/10.1080/15213269.2017.1416296.

Alhabash, S., \& Ma, M. (2017). A tale of four platforms: Motivations and uses of Facebook, Twitter, Instagram, and Snapchat among college students? Social Media+ Society, 3(1). https://doi.org/10.1177/2056305117691544.

American Psychiatric Association. (2013). Diagnostic and statistical manual of mental disorders (5th ed.). Arlington: American Psychiatric Association.

Andreassen, C. S., Billieux, J., Griffiths, M. D., Kuss, D. J., Demetrovics, Z., Mazzoni, E., \& Pallesen, S. (2016). The relationship between addictive use of social media and video games and symptoms of psychiatric disorders: A large-scale cross-sectional study. Psychology of Addictive Behaviors, 30, 252-262.

Aslam, S. (2018). Instagram by the numbers: stats, demographics \& fun facts. Retrieved January 19, 2018, from: https://www.omnicoreagency.com/instagram-statistics/

Bahtiyar, M. \& Kircaburun, K. (2017). Investigation of high school students' social media use in the context of children's rights. Paper presented at International Children's Rights Congress, Duzce, Turkey.

Balakrishnan, J., \& Griffiths, M. D. (2017). Social media addiction: What is the role of content in YouTube? Journal of Behavioral Addictions, 6(3), 364-377.

Bányai, F., Zsila, Á., Király, O., Maraz, A., Elekes, Z., Griffiths, M. D., et al. (2017). Problematic social media use: Results from a large-scale nationally representative adolescent sample. PLOS ONE, 12(1), e0169839.

Baumeister, R. F. (1990). Suicide as escape from self. Psychological Review, 97(1), 90-113.

Blackwell, D., Leaman, C., Tramposch, R., Osborne, C., \& Liss, M. (2017). Extraversion, neuroticism, attachment style and fear of missing out as predictors of social media use and addiction. Personality and Individual Differences, 116, 69-72.

Bracken, C. C. (2005). Presence and image quality: The case of high-definition television. Media Psychology, 7(2), 191-205.

Bulu, S. T. (2012). Place presence, social presence, co-presence, and satisfaction in virtual worlds. Computers \& Education, 58(1), 154-161.

Chen, G. M. (2011). Tweet this: A uses and gratifications perspective on how active Twitter use gratifies a need to connect with others. Computers in Human Behavior, 27(2), 755-762.

Coxon, M., Kelly, N., \& Page, S. (2016). Individual differences in virtual reality: Are spatial presence and spatial ability linked? Virtual Reality, 20(4), 203-212.

Chen, H. T., \& Kim, Y. (2013). Problematic use of social network sites: the interactive relationship between gratifications sought and privacy concerns. Cyberpsychology, Behavior, and Social Networking, 16(11), 806-812.

Dalgarno, B., \& Lee, M. J. (2010). What are the learning affordances of 3-D virtual environments? British Journal of Educational Technology, 41(1), 10-32.

Gao, W., Liu, Z., \& Li, J. (2017). How does social presence influence SNS addiction? A belongingness theory perspective. Computers in Human Behavior, 77, 347-355.

Griffiths, M. (2005). A 'components' model of addiction within a biopsychosocial framework. Journal of Substance Use, 10(4), 191-197.

Griffiths, M. D., Kuss, D. J., \& Demetrovics, Z. (2014). Social networking addiction: An overview of preliminary findings. In K. Rosenberg \& L. Feder (Eds.), Behavioral addictions: criteria, evidence and treatment (pp. 119-141). New York: Elsevier.

Greitemeyer, T., Mügge, D. O., \& Bollermann, I. (2014). Having responsive Facebook friends affects the satisfaction of psychological needs more than having many Facebook friends. Basic and Applied Social Psychology, 36(3), 252-258.

Hartmann, T., Klimmt, C., \& Vorderer, P. (2010). Telepresence and media entertainment. In C. C. Bracken \& P. D. Skalski (Eds.), Immersed in media: telepresence in everyday life (pp. 137-157). New York: Routledge.

Hartmann, T., Wirth, W., Schramm, H., Klimmt, C., Vorderer, P., Gysbers, A., et al. (2015). The spatial presence experience scale (SPES). Journal of Media Psychology, 28(1), 1-15.

Heeter, C. (1992). Being there: The subjective experience of presence. Presence: Teleoperators \& Virtual Environments, 1(2), 262-271.

Hu, L. T., \& Bentler, P. M. (1999). Cutoff criteria for fit indexes in covariance structural analysis: Conventional criteria versus new alternatives. Structural Equation Modelling, 6(1), 1-55. 
Huang, H. (2011). Social media addiction among adolescents in urban China: An examination of sociopsychological traits, uses and gratifications, academic performance, and social capital (Doctoral dissertation, Chinese University of Hong Kong).

Ijsselsteijn, W. A., de Ridder, H., Freeman, J., \& Avons, S. E. (2000). Presence: Concept, determinants and measurement. Paper presented at Proceedings of the SPIE, Human Vision and Electronic Imaging, San Jose, CA: International Society for Optics and Phonetics.

Kırcaburun, K. (2016a). Self-esteem, daily internet use and social media addiction as predictors of depression among Turkish adolescents. Journal of Education and Practice, 7(24), 64-72.

Kircaburun, K. (2016b). Effects of gender and personality differences on twitter addiction among Turkish undergraduates. Journal of Education and Practice, 7(24), 33-42.

Kircaburun, K., \& Griffiths, M. D. (2018). Instagram addiction and the Big Five of personality: The mediating role of self-liking. Journal of Behavioral Addictions, 7, 158-170.

Kircaburun, K., Alhabash, S., Tosuntaș, Ș.B., \& Griffiths, M.D. (2018a). Uses and gratifications of problematic social media use among university students: A simultaneous examination of the big five of personality, social media platforms and social media use motives. International Journal of Mental Health and Addiction, https://doi.org/10.1007/s11469-018-9940-6.

Kircaburun, K., Demetrovics, Z., \& Tosuntaș, Ș.B. (2018b). Analyzing the links between problematic social media use, dark triad traits and self-esteem. International Journal of Mental Health and Addiction, 1-12. https://doi.org/10.1007/s11469-018-9900-1.

Kircaburun, K., Kokkinos, C. M., Demetrovics, Z., Király, O., Griffiths, M. D., \& Çolak, T. S. (2018c). Problematic online behaviors among adolescents and emerging adults: Associations between cyberbullying perpetration, problematic social media use and psychosocial factors. International Journal of Mental Health and Addiction, https://doi.org/10.1007/s11469-018-9894-8.

Kircaburun, K. \& Bahtiyar, M. (2017). Differentiation of university students' social media use according to gender and age. Paper presented at International Instructional Technologies \& Teacher Education Symposium, Izmir, Turkey.

Kuss, D. J., \& Griffiths, M. D. (2017). Social networking sites and addiction: Ten lessons learned. International Journal of Environmental Research and Public Health, 14(3), 311.

Lee, Y. (2017). Exploring the role of escapism in the DSM-V criteria for internet gaming disorder: A metaanalytic investigation (Doctoral Dissertation, State University of New York at Albany).

Lee, E., Kim, Y. J., \& Ahn, J. (2014). How do people use Facebook features to manage social capital? Computers in Human Behavior, 36, 440-445.

Ling, Y., Nefs, H. T., Morina, N., Heynderickx, I., \& Brinkman, W. P. (2014). A meta-analysis on the relationship between self-reported presence and anxiety in virtual reality exposure therapy for anxiety disorders. PLoS ONE, 9(5), e96144.

Lombard, M., \& Ditton, T. (1997). At the heart of it all: the concept of presence. Journal of Computer-Mediated Communication, 3(2). Retrieved from http://onlinelibrary.wiley.com/doi/10.1111/j.1083-6101.1997.tb00072.x/full

Masur, P. K., Reinecke, L., Ziegele, M., \& Quiring, O. (2014). The interplay of intrinsic need satisfaction and Facebook specific motives in explaining addictive behavior on Facebook. Computers in Human Behavior, 39, $376 \mathrm{e} 386$.

Monacis, L., de Palo, V., Griffiths, M. D., \& Sinatra, M. (2017). Social networking addiction, attachment style, and validation of the Italian version of the Bergen Social Media Addiction Scale. Journal of Behavioral Addictions, 6(2), 178-186.

Murphy, D. J. (2016). Spatial presence, psychophysiology, and game(play) emotions. Social Psychology, 76(5), 805-819.

Park, S., \& Hwang, H. S. (2009). Understanding online game addiction: connection between presence and flow. In J. A. Jacko (Ed.), Human-computer interaction. Interacting in various application domains. Lecture notes in computer science (pp. 378-386). Berlin: Springer.

Poeschl, S., \& Doering, N. (2015). Measuring co-presence and social presence in virtual environments-psychometric construction of a German scale for a fear of public speaking scenario. In B. K. Wiederhold, G. Riva, \& M. D. Wiederhold (Eds.), Annual review of cybertherapy and telemedicine (pp. 58-63). Amsterdam: IOS Press.

Spada, M. M. (2014). An overview of problematic Internet use. Addictive Behaviors, 39(1), 3-6.

Satici, S. A. (2018). Facebook addiction and subjective well-being: A study of the mediating role of shyness and loneliness. International Journal of Mental Health and Addiction, 1-15. https://doi.org/10.1007/s11469-017-9862-8

Short, J., Williams, E., \& Christie, B. (1976). The social psychology of telecommunications. London: Wiley.

Slater, M., Sadagic, A., Usoh, M., \& Shroeder, R. (2000). Small-group behavior in a virtual and real environment: A comparative study. Presence:Teleoperators and Virtual Environments, 9(1), 37-51.

Stavropoulos, V., Wilson, P., Kuss, D., Griffiths, M., \& Gentile, D. (2017). A multilevel longitudinal study of experiencing virtual presence in adolescence: The role of anxiety and openness to experience in the classroom. Behaviour \& Information Technology, 36(5), 524-539.

Wegmann, E., Stodt, B., \& Brand, M. (2015). Addictive use of social networking sites can be explained by the interaction of Internet use expectancies, Internet literacy, and psychopathological symptoms. Journal of Behavioral Addictions, 4(3), 155-162. 
Westerman, D., Spence, P. R., \& Lin, X. (2015). Telepresence and exemplification in health messages: The relationships among spatial and social presence and exemplars and exemplification effects. Communication Reports, 28(2), 92-102.

Williams, K. D. (2014). The effects of dissociation, game controllers, and 3D versus 2D on presence and enjoyment. Computers in Human Behavior, 38, 142-150.

Wirth, W., Hofer, M., \& Schramm, H. (2012). The role of emotional involvement and trait absorption in the formation of spatial presence. Media Psychology, 15(1), 19-43.

Xanidis, N., \& Brignell, C. M. (2016). The association between the use of social network sites, sleep quality and cognitive function during the day. Computers in Human Behavior, 55, 121-126.

Yee, N. (2006). Motivations for play in online games. Cyberpsychology \& Behavior, 9(6), 772-775.

Young, N. L., Kuss, D. J., Griffiths, M. D., \& Howard, C. J. (2017). Passive Facebook use, Facebook addiction, and associations with escapism: An experimental vignette study. Computers in Human Behavior, 71, $24-31$. 